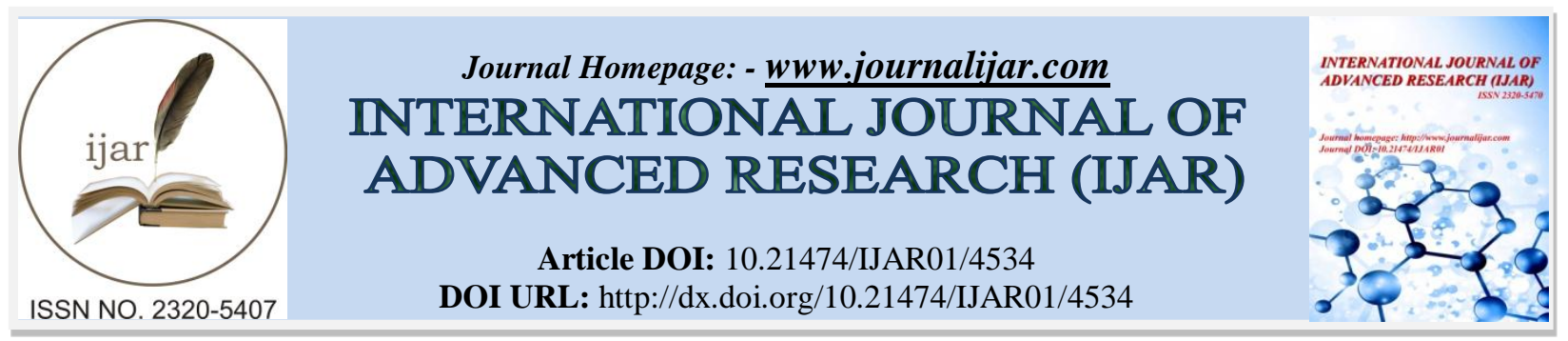

RESEARCH ARTICLE

\title{
APPLICATION OF WAVELET REGRESSION WITH LOCAL LINEAR QUANTILE REGRESSION IN FINANCIAL TIME SERIES FORECASTING.
}

\section{A. Ghazal, W. Alabeid and Gh. Alshreef.}

Department of Mathematics, Faculty of Science, Damietta University, Damietta, Egypt.

\section{Manuscript Info}

Manuscript History

Received: 19 April 2017

Final Accepted: 21 May 2017

Published: June 2017

Key words:-

Wavelet Regression, Local linear

Quantile Regression, Boundary

Problems, Bandwidth Selection.

\section{Abstract}

The classical wavelet methods suffering from boundary problems caused by the application of the wavelet transformations to a finite signal, to treatment boundary problems with wavelet regression, we propose a simple method that decreasing bias at the boundaries, it is based on a combination of wavelet functions and local linear quantile regression (WR- LLQ). We use the proposed technique to forecast stock index time series. Detailed experiments are implemented for the proposed method, in which WR- LLQ, WR, and WR-LP methods are compared. The proposed WR- LLQ model is determined to be superior to the WR and WR-LP methods in predicting the stock closing prices.

Copy Right, IJAR, 2017,. All rights reserved.

\section{Introduction:-}

Wavelet regression is a new non parametric method characterized by the ability to detect unusual appearances, which might be observed in noisy data. Tendency, collapse points, and discontinuities can be taken into consideration by wavelet methods, but when performing wavelet regression it is usual to consider some $\mathrm{f}$ boundary assumptions, such as periodicity or symmetry. However, such assumptions may not always be logical to treat this problem, it is suggested by Oh, Naveau, and Lee (2001) to split $\mathrm{f}$ as the sum of a set of wavelet basis functions, $\mathrm{f}_{\mathrm{w}}$, plus a low-order polynomial, $f_{\mathrm{P}}$. So $f=f_{\mathrm{w}}+f_{\mathrm{P}}$. The hope is that, once $\mathrm{f}_{\mathrm{P}}$ is removed from $\mathrm{f}$, the remaining portion $f_{\mathrm{w}}$ can be well estimated using wavelet regression with the said periodic boundary assumption. Practically, this approach requires choosing of the polynomial order for $\mathrm{f}_{\mathrm{P}}$ and the wavelet thresholding value for $f_{\mathrm{w}}$. Lee, Oh (2004), Naveau, and Oh (2003) propose a simple method called polynomial wavelet regression (PWR) for handling these boundary problems. Oh and Lee (2005) proposed a method for correcting the boundary bias, they join wavelet shrinkage with local polynomial regression, where the latter regression technique known of a perfect boundary properties. Simulation results from both the univariate and bivariate settings provide strong evidence that the proposed method is very successful in terms of rectify boundary bias.

Originally, this paper proposes an effective method for cure the boundary bias introduced by the incompatible of such periodic or symmetric assumption, our idea is to combine wavelet regression (WR) with local linear quantile regression (LLQ), where (LLQ) is known to possess excellent boundary properties, the proposed method consists of two stages that automatically decrease the boundary effects of wavelet method, at the first stage, LLQ which applied to the corrupted and noisy data, at the second stage, wavelet method. The final estimate is the summation of the fitting estimates from (LLQ) and (WR). Compare the results with the results obtained by Oh and Naveau (2001). The aims of this research are to study these new estimators that are combinations of local linear quantile regression 
terms and wavelet terms, (wavelet combined with local linear quantile regression) and to overcome boundary problems with wavelet regression.

\section{Wavelet Regression:-}

Mathematically we can say that wavelets are functions that break up data into distinct frequency components, and then each component is studied with a resolution matched to its scale. Wavelet analysis has been found as a powerful tool for the nonparametric estimation of spatially-variable objects, we discuss in detail wavelet methods in nonparametric regression, where the data are modeled as observations of a signal contaminated with additive Gaussian noise. Wavelet is a $\varphi$ function such as $\left\{2^{\mathrm{j}} / 2 \varphi\left(2^{\mathrm{j}}-\mathrm{k}\right) . \mathrm{j}, \mathrm{k} \in \mathrm{Z}\right\}$ that is an orthonormal basis for $\mathrm{l}^{2}(\mathrm{R})$. This $\varphi$ function is called (mother wavelet), which can be often constructed from (father wavelet). The father wavelet is not a wavelet, but we can construct wavelets from it, so it is equally important as mother wavelet.

Let $\emptyset$ and $\varphi$ be as father and a mother wavelet respectively. Any square integrable f function can be written as the following expansion (e.g., Daubechies 1992, p. 130):

$$
f_{w}(x)=\sum_{k=-\infty}^{\infty} c_{0, k} \emptyset_{k}(x)+\sum_{j=0}^{\infty} \sum_{k=-\infty}^{\infty} d_{j, k} \varphi_{j, k}(x)
$$

where $\emptyset_{k}(x)=2^{1 / 2} \emptyset(2 x-k)$ and $\varphi_{j, k}(x)=2^{j / 2} \varphi\left(2^{j} x-k\right)$.

Here the scaling and detail coefficients are respectively equal to $c_{0, k}=\int_{-\infty}^{\infty} f(x) \emptyset_{k}(x) d x$ and $d_{j, k}=\int_{-\infty}^{\infty} f(x) \varphi_{j, k}(x) d x$.

Equation (2.1) suggests the following classical nonlinear wavelet regression estimator:

$$
\hat{f}_{w}(x)=\sum_{k=1}^{2^{J}-1} \hat{C}_{0, k} \emptyset_{k}(x)+\sum_{j=0}^{\infty} \sum_{k=-\infty}^{\infty} \hat{d}_{j, k}^{s} \varphi_{j, k}(x)
$$

where $\hat{c}_{0, k}=\sum_{i} y_{i} \emptyset_{k}(i / n)$ and $\hat{d}_{j, k}=\sum_{i} y_{i} \varphi_{j, k}\left(\frac{i}{n}\right)$ are respectively the empirical scaling and detail coefficients, and $\hat{d}_{j, k}^{s}=\operatorname{sgn}\left(\hat{d}_{j, k}\right) \max \left(0,\left|\hat{d}_{j, k}\right|-\lambda\right.$.

Sometimes the soft-thresholded coefficients $\hat{d}_{j, k}^{s}$ are replaced by the hard-thresholded coefficients $\hat{d}^{H}{ }_{j, k}=$ $\hat{d}_{j, k} I_{\left(\left|\hat{d}_{j, k}\right|>\lambda\right)}$ (see, for examples, Donoho and Johnstone $\left.(1994,1995)\right)$.

\section{Boundary Problems:-}

In order to overcome the boundaries problems when using wavelet regression, two types of approach are usually used. The first one can either put some extra constraints on the function $\mathrm{f}$, such as periodicity, symmetry or anti symmetry, second one construct some specific wavelets on $\mathrm{l}^{2}[0,1]$.

The advantage of the first method is that it is easy to be applied to real data. Indeed, wavelet code for most highlevel statistical or mathematical software packages, like S-Plus or R, are now accessible, then, artificially large wavelet coefficients result when the extra constraints on $\mathrm{f}$ are not satisfied, and artificial wiggles are created at the boundaries.

The second method is to construct specific wavelet functions on $\mathrm{l}^{2}[0,1]$. One difficulty is that inquiry a modified discrete wavelet transform is considerably more embroiled than implementing the classical transform and imposing the simple periodic or symmetric boundary conditions.

Objective in this paper is to provide a new approach that is easily to be carrying out and that also has the power to significantly minimize the bias at boundaries observed with periodic or symmetric boundary conditions

\section{Local Linear Quantile (LLQ) Regression:-}

Basically, the seminal study of Koenker and Bassett [1978] introduced parametric quantile regression, which is considered an alternative to the classical regression in both parametric and nonparametric fields. Numerous models 
for the nonparametric approach have been introduced in statistical literature, such as the locally polynomial quantile regression by Chaudhuri (1991) and the kernel methods by Koenker et al. [1994]. In this paper, we endorse the LLQ regression proposed by Yu and Jones (1998).

Let $\left\{\left(x_{i}, y_{i}\right), i=1 \ldots, n\right\}$ be bivariate observations. To estimate the $\tau$ th conditional quantile function of response $y$, the equation below is defined given $\mathrm{X}=\mathrm{x}$ :

$$
g(x)=Q_{y}(\tau \mid x)
$$

We will put $\mathrm{K}$ as positive symmetric unimodal kernel function, and consider the following weighted quantile regression problem:

where $w(x)=k\left(\left(x_{i}-x\right) / h\right) / h$.

$$
\frac{\min }{\beta \in i^{2}} \sum_{i=1}^{n} w_{i}(x) \rho_{\tau}\left(y i-\beta_{0}-\beta_{1}\left(x_{i}-x\right)\right),
$$

Once the covariate observations are centered at point, the estimate of $g(x)$ is simply $\beta_{0}$, which is the first component of the minimizer of (3.1) and determines the estimate of the slope of the function $g$ at point $x$.

\section{Proposed Method:-}

The proposed method consists of two stages that automatically decrease the boundary effects of wavelet method, at the first stage, LLQ which is considered as an excellent boundary treatment Cai and Xu (2008) is applied to the corrupted and noisy data. The remaining series is then expected to be hidden in the residuals, at the second stage, wavelet method is applied to the residuals. The final estimate is the summation of the fitting estimates from LLQ and WR. Compare the results with the results obtained by Oh and Naveau (2001).

The main idea of the proposed method is to estimate the underlying function $f$ with the sum of a set of wavelet regression functions, $\mathrm{f}_{\mathrm{w}}$, and LLQ function, $\mathrm{f}_{\mathrm{LLQ}}$. That is,

$$
\hat{f}_{W \cdot L L Q}=\hat{f}_{w}+\hat{f}_{L L Q}
$$

To obtain the wavelet regression - Local Linear Quantile Regression estimate $\hat{\mathrm{f}}_{\mathrm{W} . \mathrm{LLQ}}$ we need to estimate the two components: $\hat{f}_{w}$ and $\hat{f}_{\text {LLQ }}$. Inspired by the back-fitting algorithm of Hastie and Tibshirani (1990), we propose the following iterative algorithm for computing $\hat{\mathrm{f}}_{\mathrm{w}}, \hat{\mathrm{f}}_{\mathrm{LLQ}}$ and hence $\hat{\mathrm{f}}_{\mathrm{W} . \mathrm{LLQ}}$.

1. Obtain an initial estimate $\hat{f}^{0}$ for $f$, and set $\hat{f}_{L L Q}^{0}=\hat{f}^{0}$.

2. For $j=1, \ldots .$. , iterate the following steps:

(a) Apply wavelet regression to $y_{i}-\hat{f}_{L L Q}^{J-1}$ and obtain $\hat{f}_{W}^{J}$.

(b) Estimate $\hat{f}_{L L Q}^{J}$ by fitting local quantile regression to $y_{i}-\hat{f}_{w}^{J}$

3. Stop if $\hat{f}_{W \cdot L L Q}=\hat{f}_{L L Q}^{J}+\hat{f}_{w}^{J}$ converges.

To use the above algorithm, one needs to choose the initial curve estimate $\hat{\mathrm{f}}^{0}$ in Step 1 and the smoothing parameter for the local quantile fit $\hat{\mathrm{f}}_{\mathrm{LLQ}}^{\mathrm{J}}$ in Step 2(b). For computing $\hat{\mathrm{f}}^{0}$, we use high-level statistical or mathematical software packages $(\mathrm{R})$..

\section{Bandwidth Selection:-}

The practical performance of $\widehat{Q}_{\mathrm{x}}(\mathrm{x})$ strongly depends on the selected bandwidth parameter. We take on the strategy of $\mathrm{Yu}$ and Jones [1998]. In sum, we will employ the automatic bandwidth selection strategy for smoothing conditional quantiles as follows. 
1- We will use the ready-made and sophisticated methods in selecting $h_{\text {mean }}$; we employ Ruppert, Sheather, and Wand (1995) who explored a "direct plugin" bandwidth selection procedure, which relies on asymptotically optimal bandwidth

$$
\begin{array}{r}
\mathrm{h}_{\text {mean }}=\left[\sigma^{2} \mathrm{R}(\mathrm{k})(\mathrm{b}-\mathrm{a}) / \mathrm{n} \mu_{2}^{2} \int\left\lfloor\left.\left.\mathrm{m}_{(\mathrm{x})}^{\prime \prime}\right|^{2} \mathrm{p}_{(\mathrm{x})} \mathrm{d}(\mathrm{x})\right|^{1 / 5}\right.\right. \\
=\mathrm{c}_{1}(\mathrm{k})\left\{\frac{\sigma^{2}(\mathrm{~b}-\mathrm{a})}{\mathrm{n} \theta_{22}}\right\}^{1 / 5}
\end{array}
$$

2- We use $h_{\tau}=h_{\text {mean }}\left\{\tau(1-\tau) /\left(\varnothing\left(\Phi^{-1}(\tau)\right)^{2}\right\}^{1 / 5}\right.$ to obtain all of the other $h_{\tau}$ from $h_{\text {mean }}$. where $\emptyset$ and $\Phi$ are standard normal density and distribution functions, and $\mathrm{h}_{\text {mean }}$ is a bandwidth parameter for regression mean estimation with various existing methods. This procedure obtains identical bandwidths for the $\tau$ and $(1-\tau)$ quantiles.

\section{Description of The data and Experiment Analysis:-}

The daily closing prices of major European stock indices, 1991-1998: Germany DAX (Ibis), and UK FTSE. The data are sampled in business time, i.e., weekends and holidays are omitted, a multivariate time series with 1860 observations on 4 variables. The data were kindly provided by Erste Bank AG, Vienna, Austria.

Presents the proposed method for improving boundary adjustment in wavelet regression. Driven by the fact that local quantile regression is extremely effective in adapting to boundary conditions. We analyze the indices based on the WR-LLQ and WR and the WR-LP methods.

The forecasting accuracy measures employed in this study are root mean square error (RMSE), mean absolute error (MAE), and mean absolute percentage error (MAPE). The RMSE, MAE, and MAPE values obtained through the WR -LLQ, WR and WR-LP methods. In each test set for the two index series are summarized in Tables 1 and 2. The results demonstrate that the proposed WR -LLQ method is more successful in all cases in forecasting the stock closing prices than the WR and the WR-LP methods.
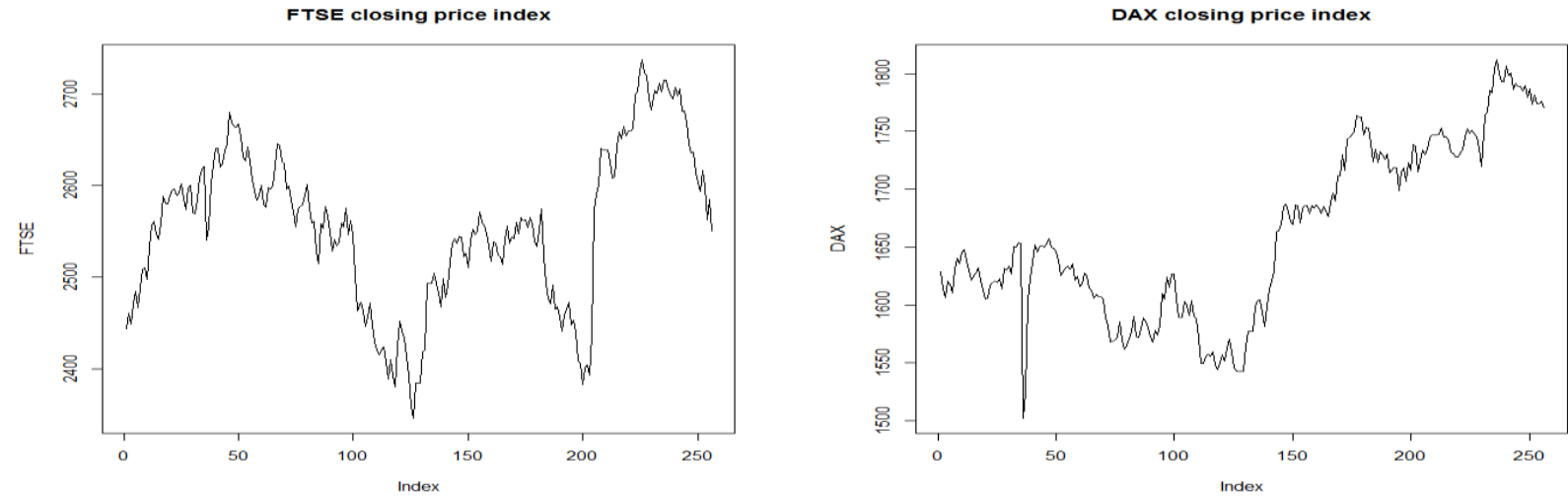

Figure 1:- FTSE price and DAX closing price index, respectively.

Table 1:- Comparison of RMSE, MAE, and MAPE values for DAX using the WR-LP, WR, and WR-LLQ methods

\begin{tabular}{|l|l|l|l|l|}
\hline quantiles & n.head=10 & RMSE & MAE & MAPE \\
\hline \multirow{4}{*}{$\tau_{0.50}$} & WR & 16.0152 & 14.3811 & 0.817226 \\
\cline { 2 - 5 } & WR-LP & 13.00762 & 11.23252 & 0.638528 \\
\cline { 2 - 5 } & WR-LLQ & 9.033317 & 7.978742 & 0.45279 \\
\hline \multirow{3}{*}{$\tau_{0.75}$} & WR & 16.0152 & 14.3811 & 0.817226 \\
\cline { 2 - 5 } & WR-LP & 13.00762 & 11.23252 & 0.638528 \\
\cline { 2 - 5 } & WR-LLQ & 8.294448 & 7.277219 & 0.412813 \\
\hline \multirow{3}{*}{$\tau_{0.95}$} & WR & 16.0152 & 14.3811 & 0.817226 \\
\cline { 2 - 5 } & WR-LP & 13.00762 & 11.23252 & 0.638528 \\
\cline { 2 - 5 } & WR-LLQ & 11.67783 & 8.983242 & 0.507962 \\
\hline
\end{tabular}


Table 2:- Comparison of RMSE, MAE, and MAPE values for FTSE using the WR-LP, WR, and WR-LLQ methods

\begin{tabular}{|l|l|l|l|l|}
\hline quantiles & n.head=10 & RMSE & MAE & MAPE \\
\hline \multirow{3}{*}{$\tau_{0.50}$} & WR & 130.026 & 123.2905 & 4.924095 \\
\cline { 2 - 5 } & WR-LP & 62.7433 & 55.62809 & 2.226658 \\
\cline { 2 - 5 } & WR-LLQ & 22.84248 & 18.69655 & 0.748319 \\
\hline \multirow{3}{*}{$\tau_{0.75}$} & WR & 130.026 & 123.2905 & 4.924095 \\
\cline { 2 - 5 } & WR-LP & 62.7433 & 55.62809 & 2.226658 \\
\cline { 2 - 5 } & WR-LLQ & 42.87112 & 36.72086 & 1.471088 \\
\hline \multirow{2}{*}{$\tau_{0.95}$} & WR & 130.026 & 123.2905 & 4.924095 \\
\cline { 2 - 5 } & WR-LP & 62.7433 & 55.62809 & 2.226658 \\
\cline { 2 - 5 } & WR-LLQ & 49.55608 & 41.91537 & 1.679824 \\
\hline
\end{tabular}

\section{Conclusions:-}

In this paper a wavelet regression local quantile regression method is proposed to minimize the boundary bias that is commonly found in the wavelet method, whereas the proposed method is based on a coupling of classical the wavelet method and local quantile regression. The performance of the method was tested on different real data, results from these experiments illustrate the improvement of the wavelet regression- local quantile regression over the classical wavelet methods.

\section{References:-}

1. Anestis Antoniadis. (2007) Wavelet methods in statistics: Some recent developments and their applications, Journal reference Statistics Surveys 2007, Vol. 1, 16-55.

2. Cai Z. and Xu X. (2008) Nonparametric quantile estimations for dynamic smooth coefficient models. Journal of the American Statistical Association, vol. 103, no. 484, pp. 1595-1608.

3. Chaudhuri P. (1991) Nonparametric estimates of regression quantiles and their local Bahadur representation. The Annals of Statistics, vol. 19, no. 2, pp. 760-777.

4. Daubechies, I. (1992) Ten Lectures on Wavelets. CBMS-NSF Regional Conference Series in Applied Mathematics. Society for Industrial and Applied Mathematics, Philadelphia, PA: SIAM

5. Donoho, D. L. and Johnstone, I. M. (1994) Ideal spatial adaptation by wavelet shrinkage. Biometrika, $81425-$ 455. MR1311089

6. Donoho D.L. and Johnstone I.M. (1995) Adapting to unknown smoothness via wavelet shrinkage. Journal of the American Statistical Association 90: 1200-1224.

7. Hastie T., Tibshirani R, (1990) Generalized Additive Models. Chapman \& Hall, London.

8. Koenker R. and Bassett G. Jr. (1978) Regression quantiles. Econometrica, vol. 46, no. 1, pp. 33-50.

9. Koenker R., Ng P., and Portnoy S. (1994) Quantile smoothing splines. Biometrika, vol. 81, no. 4, pp. 673-680.

10. Lee T.C.M. and Oh H.-S. (2004) Automatic polynomial wavelet regression, Statistics and Computing 14: 337.

11. Meyer Y. (1993) Wavelets-Algorithms and applications. Wavelets-Algorithms and applications Society for Industrial and Applied Mathematics Translation., 142.

12. Naveau P. and Oh H.-S. (2003) Polynomial wavelet regression for images with irregular boundaries. IEEE Trans. Image Process.

13. Oh H.-S., Naveau, P. and Lee, G. (2001) Polynomial boundary treatment for wavelet regression. Biometrika $88,291-298$.

14. Oh H.-S. and Lee T. C. M.( 2005) Hybrid local polynomial wavelet shrinkage: wavelet regression with automatic boundary adjustment. Computational Statistics \& Data Analysis, vol. 48, no. 4, pp. 809-819.

15. Ruppert D., Sheather S. J. and M. P. Wand (1995) An effective bandwidth selector for local least squares regression. Journal of the American Statistical Association, vol. 90, no. 432, pp. 12571270.

16. Yu K. and Jones M. C.(1998) Local linear quantile regression. Journal of the American Statistical Association, vol. 93, no. 441, pp. 228- 237. 